\title{
Optical Analysis of Direct Laser Written Structures
}

\author{
Xiang Liu ${ }^{1}$, Patrick Salter ${ }^{1}$ and Martin Booth ${ }^{1,2}$ \\ ${ }^{1}$ Department of Engineering Science, University of Oxford, Parks Road, Oxford OX1 3PJ, United Kingdom \\ ${ }^{2}$ Centre for Neural Circuits and Behaviour, Tinsley Building, Mansfield Road, Oxford, OXI 3SR, United Kingdom \\ xiang.liu@eng.ox.ac.uk
}

\begin{abstract}
To assess the quality of waveguides that can be made by ultrafast laser direct writing, consistent waveguide characterisation methodologies need to be constructed. We aim to develop a suite of complementary optical techniques for such a task, including measurements of structure, morphology and refractive index profile as appropriate. We employ confocal and harmonic generation microscopes in addition to phase tomography.
\end{abstract}

\section{Introduction}

There is significant interest in Direct Laser Writing (DLW) of photonic waveguides in bulk glasses using ultrafast pulsed lasers [1]. Void structures or local refractive index increase can be generated under tight spatial focusing. Optical waveguides are then formed by translating the substrate relative to the beam. By coupling multiple waveguides together, complex photonic circuits can be fabricated. The applications of this technology are both broad and highly promising [2].

One of the challenges facing the DLW technology is the development of a method for complete characterisation of the micromachined structures. The relatively weak refractive index contrast introduced in waveguide fabrication is difficult to measure both in magnitude and morphology, although it is an important parameter for predicting propagation and coupling losses, and mode profiles. Parallel processing techniques have been proposed and demonstrated to increase the fabrication speed for large devices [3-7]. However, this renders the characterisation techniques highly important as uniformity of the multiple focal spots cannot be guaranteed by such parallel processing. For holographic parallel methods, this non-uniformity is in part due to the inherent off-axis chromatic aberration. Therefore a well developed characterisation method would provide in situ calibration of structures produced by parallel laser processing. A great advantage of the DLW technique when compared with traditional fabrication methods is that it creates three-dimensional structures. It is therefore essential that the characterisation methods provide three-dimensional resolution. In order to characterise structures at different depths within the substrate, we should also compensate depth dependent spherical aberration.

We have developed a suite of methods to analyse the structural properties of fabricated photonic devices. We have obtained information about the refractive index modulation, cross-section profile and three-dimensional structure, which provides a better understanding of the fabrication process. These techniques can be developed for online monitoring of micromachining.

\section{Confocal and third harmonic generation microscope measurements}

Scanning reflection confocal microscopy is the first method we have implemented. The measured signal arises from reflections at boundaries in refractive index and scatter from void structures and defects. A confocal detection pinhole is used to obscure out-of-focus light, thus revealing the three dimensional structure of fabricated features[8]. This three dimensional resolution is similar to that of our recently demonstrated third harmonic generation (THG) microscope, but achieved by a simpler optical system [9]. A deformable mirror is incorporated to correct aberrations encountered in imaging [10,11]. Figure 1 (a) shows a confocal microscope characterisation of the defects fabricated in fused silica, while Figure 1 (b) and (c) show waveguide cross-sections from a THG microscope.

\section{Measurement of refractive index}

With the confocal and THG microscopes we can characterise the 3D nature of fabricated structures. It would also be useful, particularly for waveguide analysis, to develop a simple optical technique for measurement of the refractive index contrast for the structures. Here we implement the transfer of intensity approach [12,13], where we can calculate the phase change from measurements of transmitted light intensity at different defocus planes. This can be implemented in 2D live during the fabrication process, and we explore whether off-line tomographic measurements can give further information on the 3D refractive index profile. 
(a)
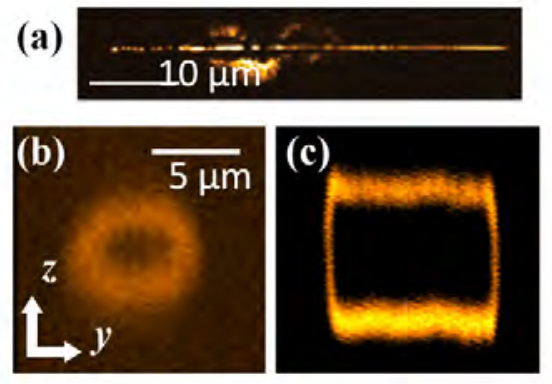
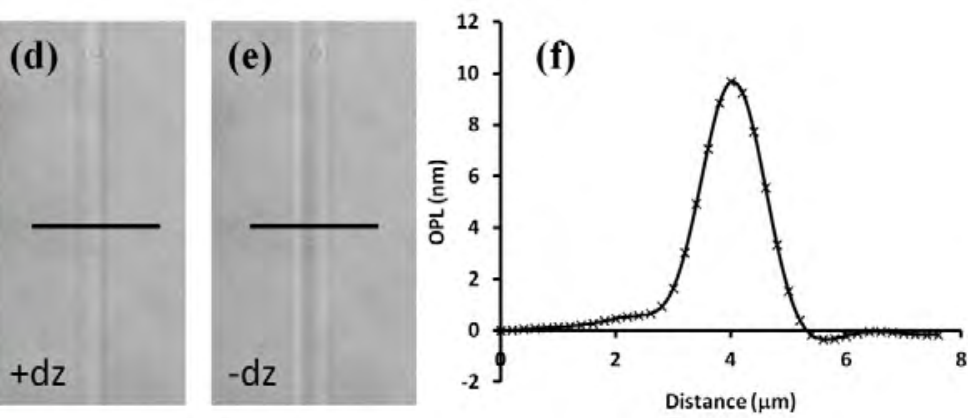

Figure 1 a) Reflection confocal image of void structures in fused silica. (b) and (c) third harmonic generation (THG) images of waveguides with respective circular and square cross-sections. (d) and (e) brightfield transmission images of a waveguide at two defocus planes which may be used to calculate the associated phase retardation along the line indicated (f).

\section{References}

[1] R. R. Gattass and E. Mazur, "Femtosecond laser micromachining in transparent materials," Nat. Photon., 2, 219 (2008).

[2] G. Della Valle, R. Osellame and P. Laporta, "Micromachinning of photonic devices by femtosend laser pulses,” J. Opt. A, 11, 013001 (2009).

[3] A. Jesacher and M. Booth, "Parallel direct laser writing in three dimensions with spatially dependent aberration correction," Opt. Exp., 18, 21090-21099 (2010).

[4] J. -I. Kato, N. Takeyasu, Y. Adachi, S. Kawata and H. -B. Sun, "Multiple-spot parallel processing for laser micro-nano fabrication," Appl. Phys. Lett., 86, 044102 (2005).

[5] Y. Kuroiwa, N. Takeshima, Y. Narita, S. Tanaka and K. Hirao, "Arbitrary micropatterning method in femtosecond laser microprocessing using diffractive optical elements," Opt. Exp., 12, 1908-1915 (2004).

[6] S. Hasegawa, Y. Hayasaki and N. Nishida, "Holographic femtosend laser processing with multiplexed phase Fresnel lenses," Opt. Lett., 31 , 1705-1707 (2006).

[7] M. Yamaji, H. Kawashima, J. Suzuki and S. Tanaka, "Three dimensional micromaching inside a transparent material by single pulse femtosecond laser through a hologram,” Appl. Phys. Lett., 93, 041116 (2008).

[8] A. Jesacher, G. D. Marshall, T. Wilson and M. J. Booth, "Adaptive optics for direct laser writing with plasma emission aberration sensing," Opt. Exp., 18, 656 (2010).

[9] G. D. Marshall, A. Jesacher, A. Thayil, M. J. Withford and M. Booth, "Three-dimensional imaging of direct-written photonic structures," Opt. Lett., 36, 695 (2011).

[10] P. Salter, A. Jesacher, R. Simmonds and M. Booth, “Aberration correction in laser micro-fabrication,” ALIU: The Laser User, 64, 28 (2011).

[11] P. S. Salter, A. Jesacher, J. B. Spring, B. J. Metcalf, N. Thomas-Peter, R. D. Simmonds, N. K. Langford, I. A. Walmsley and M. J. Booth, "Adaptive slit beam shaping for direct laser written waveguides," 37, 470 (2012).

[12] D. Paganin and K. A. Nugent, "Noninterferometric Phase Imaging with Partially Coherent Light," Phys. Rev. Lett., 80,2586 (1998).

[13] P. Masselin, L. D. Coq and E. Bychkov, "Refractive index variation induced by femtosecond laser direct writing in the bulk of As2S3 glas at high repetition rate," Opt. Mat., 33, 872 (2011). 